\title{
Introduction to Chern-Simons Theory
}

\author{
Ademola Adeifeoba ${ }^{\dagger}$ \\ ${ }^{\dagger}$ Institute for Theoretical Physics, \\ Heidelberg, Germany. \\ E-mail: adeifeoba@thphys.uni-heidelberg.de
}

Abstract: The $2+1$ Yang-Mills theory allows for an interaction term called the Chern-Simons term. This topological term plays a useful role in understanding the field theoretic description of the excitation of the quantum hall system such as Anyons. While solving the non-Abelian Chernsimons theory is rather complicated, its knotty world allows for a framework for solving it. In the framework, the idea was to relate physical observables with the Jones polynomials. In this note, I will summarize the basic idea leading up to this framework. 


\section{Contents}

0 Prologue 2

1 Introduction $\quad 2$

2 Elements of Anyon Models $\quad 4$

2.1 Particle Type 4

2.2 Fusion Rule 4

2.3 Artin's braid group 6

3 Chern-Simons Theory $\quad 8$

3.1 Classical CS Invariant $\quad 8$

3.2 CS Model of Anyons 8

4 Wilson Loops and Anyonic Statistics $\quad 12$

4.1 Abelian and non-Abelian $\quad 12$

4.2 Expectation Value 13

5 The Jones Polynomial $\quad 16$

$\begin{array}{lll}5.1 \text { Knots and Links } & 16\end{array}$

6 Non-Abelian CS theory $r$

6.1 Quantization on $\Sigma \times \mathbb{R} \quad 19$

6.1.1 Quantization on $\Sigma$ with punctures 20

6.2 Evaluation by TQFT 21

6.2.1 Unknotting Procedure 22 


\section{Prologue}

This note is a report in partial satisfaction of my study and seminar work on "Physical Applications of Topological Quantum Field Theory" at the University of Heidelberg. It is fair to declare forward that, while I have made efforts to carefully craft the basic ideas I have tried understood about a very fascinating topic within a very limited time, there are chances of omission or partial coverage of some concepts. I tried as much to cite important references where I consider it needful.

We shall study Witten's original paper on "Quantum field theory and the Jones polynomial." [1]. Elementary readers like me will agree that without some formal background understanding, this article belong to a class usually tagged "hard to read", more because it assumes many important advances, not only in physics but also in mathematics, as prerequisite. As such, I consider a short introduction about few background stories as useful and motivating towards our study. Following that, I will discuss the important elements of model description of anyons. The features introduced will play important roles throughout the remaining sections. I will follow similar structure of the lecture, but more explicit in this note. Our final destination is the evaluation of Wilson link invariant of the non-Abelian Chern-simon theory. The aim is to derive the rule for unknotting the Wilson link and compute the expectation value by relating physical observables to Jones polynomials which are knots invariant. As such, the roads to our final destination demands few important branches to transit towards an expected end.

\section{Introduction}

One of the ideas that revolutionized 20th century physics is Quantum field theory - a theoretical framework for modeling quantum mechanical system of elementary particles. Basically, the world is quantum, classicality is a certain limit of it and fields are nothing but part of reality. The emergent particles are excitation of fields. While in classical mechanics, particles are considered as distinguishable, new features arise on transition into Quantum theory. Among them is the fact that identical particles are indistinguishable. As a consequence, interchanging particles in a multiparticle state does not lead to a new configuration of the system, so that all probabilities is the same under such operation, i.e.

$$
\left|\psi\left(\pi\left(\mathbf{r}_{1}, \ldots, \mathbf{r}_{n}\right)\right)\right|^{2}=\left|\psi\left(\mathbf{r}_{1}, \ldots, \mathbf{r}_{n}\right)\right|^{2},
$$

where $\pi$ is the permutation operation of $n$ particle co-ordinates. More precisely, the wavefunction is left invariant by the interchanging operation up to a certain phase. This understanding led to quantum statistical decription of particles, where interchanging operation in three or higher spatial dimension leads to an interpretation of wavefunction as symmetric or antisymmetric under exchange depending on whether it transforms as boson or fermion in the following sense:

$$
\psi\left(\mathbf{r}_{1}, \mathbf{r}_{2}\right) \longrightarrow \eta \psi\left(\mathbf{r}_{2}, \mathbf{r}_{1}\right) \longrightarrow \eta^{2} \psi\left(\mathbf{r}_{1}, \mathbf{r}_{2}\right), \quad \eta= \begin{cases}+1, & \text { boson } \\ -1, & \text { fermion } .\end{cases}
$$

The study of planar physics is not a new thing in physical study. In particular, the $2 \mathrm{D}$ physics received some attention and rapid progress in the 70-90s towards understanding of possible particle statistics and their behaviour. On the first hand is an experimental physics leading to the discovery of fractional quantum Hall effect[2]. Way back in 1879, Edwin Hall had discovered the classical 
Hall effect as a result of the motion of charged particles in a magnetic field[3]. An interesting fact about the very fractional quantum Hall effect is that, the hall conductivity

$$
\sigma_{x y}=\frac{e^{2}}{2 \pi \hbar} \nu, \quad \nu \in \mathbb{Q}
$$

is surprisingly quantized. On the other hand is a theoretical curiousity leading to the prediction of quasiparticles and quasiholes whose statistics are different from those of bosons and fermions. The major stages include the following:

- Laughlin Wavefunction: This wavefunction was proposed as an ansatz to the lowest Landau levels at filling fraction $\nu=\frac{1}{m}$ for $m \in \mathbb{Z}$, with the excitations taking fractional charge $\frac{e}{m}[4]$.

- Statistics: In a system restricted to two spatial dimensions, some emergent phenomena could be observed [5-8], namely, emergent particles turns out to obey statistics interpolating between Fermi-Dirac and Bose-Einstein. These fractionally charged quasiparticle excitations of the Laughlin state are called Abelian Anyons or simply Anyons. The excitations emerge with fractional statistical angle $\theta=\frac{\pi}{m}$ as their wavefunctions acquire phase factor of $\eta=$ $e^{i \theta}$ when identical quasiparticles undergo an exchange operation similar to (1.2). When exchange operation which transforms the system's quantum state is not commutative, nonAbelian anyons arise. Examples of non-Abelian quasiparticle statistics are found in system of Fibonacci anyons and Ising anyons.

- Hierarchy states: The idea that a more generic category of fractional quantum hall effect defined by infinite continued filling fraction

$$
\nu=\frac{1}{m \pm \frac{1}{\widetilde{m_{1} \pm \frac{1}{\tilde{m}_{2} \pm \ldots}}}}
$$

was proposed and later validated quantitatively. $[6,9]$

The field theoretic framework underlying this planar physics phenomena is known as Chern-simons theory (CS). It is a topological quantum field theory (TQFT) featuring topological invariant observables. The field theory enjoys general covariance since there is no a priori choice of metric on the manifold on which the theory is formulated. As we shall see furthermore, the anyonic phenomena does not emerge from this theory as a result of gauge invariance, but rather as a consequence of the theory being quasi-invariant, i.e. while the action is not fully gauge invariant, the observables and therefore the partition function are fully gauge invariant.

Remarkably, the nature of anyons has inspired some practical purposes such as in topological quantum computation. The very features of Chern-Simons invariants have lead to new developments in mathematics. One of our goals in this study is to understand how quantum Chern-Simons invariant is related to the Jones invariants of link which have found useful applications in knot theory. 


\section{Elements of Anyon Models}

"Since interchange of two of these particles can give any phase, I will call them generically anyons." - Frank Wilczek in [8]

There is a general structure that all non-Abelian anyon models are required to feature. In this section, our goal is to discuss these basic features characterizing a model description of anyon. The features arise as a consequence of the following insights:

- Anyons can be created or annihilated pairwisely;

- Anyons can be fused to form composite anyons;

- Anyons has braiding rules.

These features will play roles in the model description of anyons by the planar Chern-Simons theory.

\subsection{Particle Type}

A non-trivial anyonic system generally consists of multiple types of anyon. Therefore, to define an anyon model, we must declare all the distinct type of anyon in the model. A given anyon model is expected to have:

- a finite set of elementary particle specie, say, $\left\{\phi_{a}, \phi_{b}, \phi_{c}, \ldots\right\}$. Each particle is locally distinguished by its label $a, b, c, \ldots$ which we think of as topological charges. These topological charges are conserved quantum numbers.

- trivial particle $\phi_{\mathbb{1}}$ which corresponds to a unique vacuum.

- antiparticle $\phi_{\bar{a}}$ such that $\phi_{a} \times \phi_{\bar{a}}=\phi_{\mathbb{1}}=\phi_{\overline{\mathbb{1}}}$. This implies that particles are only created in pair from the vacuum.

The simplest non-trivial anyon model is therefore spanned by the particle set

$$
\Xi=\left\{\phi_{\mathbb{1}}, \phi_{a}, \phi_{b}, \phi_{c}, \ldots\right\}
$$

\subsection{Fusion Rule}

Given that anyons come in multiple, bound states could exist in principle. Even when no stable bound state exists, composite anyon can be formed by bringing two anyons close together. The fusion rule of Abelian anyon and the quantum number of the eventual composite particles is straightforward. There is only one possible fusion channel. For example, fusion of two given Abelian anyons with statistic $\frac{\alpha^{2} \pi}{m}$ and $\frac{\beta^{2} \pi}{m}$ is given as

$$
\frac{\alpha^{2} \pi}{m} \times \frac{\beta^{2} \pi}{m}=\frac{(\alpha+\beta)^{2} \pi}{m} .
$$

What is the statistical behaviour of composite non-Abelian anyon? Tensor product doesn't come in handy. The answer to this arise by recognizing that topological quantum numbers combine in non-unique manner. This means that there are different possible fusion channels. Fusion rule is given as

$$
\phi_{a} \times \phi_{b}=\mathcal{N}_{a b}^{c} \phi_{c}, \quad \mathbb{Z} \ni \mathcal{N}_{a b}^{c}= \begin{cases}1, & \text { Abelian anyons } \\ \geq 2, & \text { non }- \text { Abelian anyons } .\end{cases}
$$


Fusion is depicted in figure 1.The integer number $\mathcal{N}_{a b}^{c}$ is the number of distinguishable fusion channels. These fusion channels can be understood to be orthonormal basis states

$$
\left\{|a b, c ; \mu\rangle, \quad \mu=1,2, \ldots, \mathcal{N}_{a b}^{c}\right\}
$$

of the fusion Hilbert space $\mathcal{V}_{a b}^{c}$ with the following properties:

- orthonormality: $\langle a b, c ; \mu \mid a b, d ; \nu\rangle=\delta_{c d} \delta_{\mu \nu}$

- completeness:

$$
\sum_{\mu, c}|a b, d ; \mu\rangle\langle a b, c ; \mu|=I_{a b},
$$

where $I_{a b}$ is the trivial fusion channel.

- For non-Abelian anyons,

$$
\operatorname{dim}\left(\bigoplus_{c} \mathcal{V}_{a b}^{c}\right)=\sum_{c} \mathcal{N}_{a b}^{c} \geq 2
$$

- fusion is associative, i.e.

$$
\left\{\begin{array}{l}
\phi_{a} \times \phi_{b}=\phi_{b} \times \phi_{a} \\
\left(\phi_{a} \times \phi_{b}\right) \times \phi_{c}=\phi_{a} \times\left(\phi_{b} \times \phi_{c}\right) .
\end{array}\right.
$$

This basically insist that the order of tensor product is irrelevant, so that one order of fusing three anyons can be rotated into another in the fusion space by the Fusion matrix $\mathcal{F}_{a b c}^{d}$ as described in figure 2 .

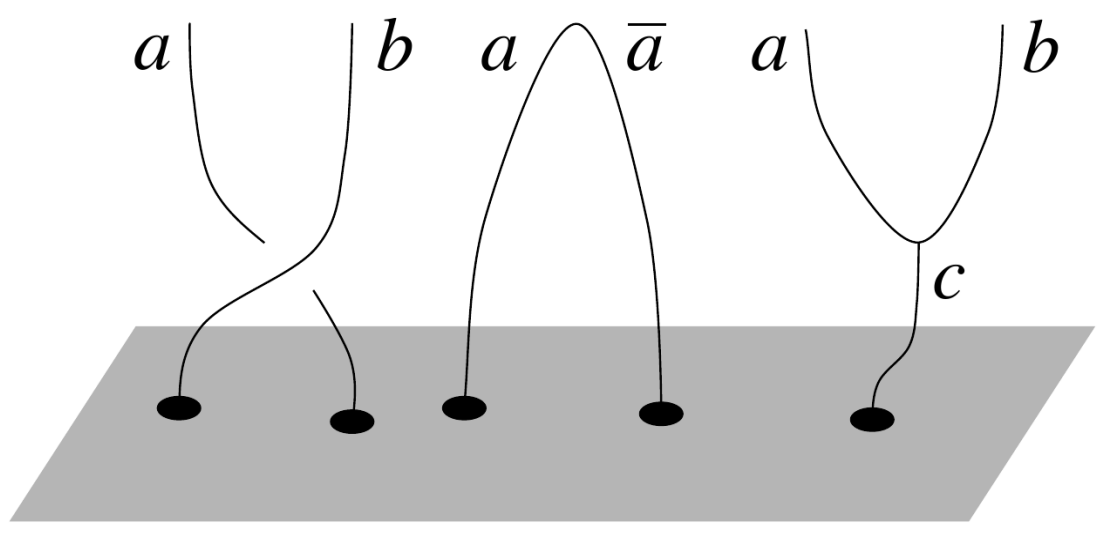

Figure 1. Worldlines of anyons. The first diagram features anyonic fundamental braid. Second diagram depicts pair-creation from the vacuum of a particle $a$ and its antiparticle $\bar{a}$. The third describes the fusion of two particles $a$ and $b$ by two lines fusion into composite $c$

The simplest model of anyonic system is Fibonacci anyons[11] . It is an anyonic system having only two particle types: the vacuum $\phi_{\mathbb{1}}$ and the nontrivial particle type $\phi_{\tau}$. Fibonacci anyons are self-dual, i.e. particle $\tau$ is the same as its anti-particle. Fusion rules just go by

$$
\left\{\begin{array}{l}
\phi_{\tau} \times \phi_{\mathbb{1}}=\phi_{\mathbb{1}} \times \phi_{\tau}=\phi_{\tau} \\
\phi_{\tau} \times \phi_{\tau}=\phi_{\mathbb{1}} \oplus \phi_{\tau},
\end{array}\right.
$$

implying that the dimension of fusion Hilbert space is 2 . 



Figure 2. Order of fusion mapped by sufficient number of moves implemented by Fusion matrix $\mathcal{F}_{a b c}^{d}$. Each order of fusion corresponds to a choice of basis with $\mathcal{F}$ matrix as a transformation between different bases. That $i$ and $j$ are different is consistent with having a fixed composite fusion outcome $d$.

\subsection{Artin's braid group}

In the introduction, we recognised in (1.1) that under the exchange of particles in $3+1$ spacetime dimensions, wavefunction describing a system of identical particles acquires \pm phase and therefore exhibits two possible types of symmetry depending on whether it is a boson or a fermion. In path integral interpretation, twice operation of particle exchange is equivalent to a process in which one of the particles is taken along a trajectory that wrap around the other. Different trajectories belong to topological class corresponding to the elements of the permutation group $S_{N}$ specifying how the initial positions are permuted into the final positions. These trajectories in three spatial dimensions can be continuously deformed into straight in time direction. As such, the wave function is left invariant under exchange operation.

We further learnt that new statistical behaviour emerges in $2+1$ spacetime dimensions, and anyons can exist in principle. The precise statement is that, under twice operation of particle exchange, $\eta^{2}$ isn't necessarily equals 1 , so that the wavefunction need not return to the initial state, i.e. it acquires a non-trivial phase such that

$$
\psi\left(\mathbf{r}_{1}, \mathbf{r}_{2}\right) \longrightarrow e^{i \theta} \psi\left(\mathbf{r}_{2}, \mathbf{r}_{1}\right) \longrightarrow e^{i 2 \theta} \psi\left(\mathbf{r}_{1}, \mathbf{r}_{2}\right),
$$

and the special case of boson and fermions are realized for $\theta=0, \pi$ respectively. Particles with statistical angle $\theta$ interpolating between 0 and $\pi$ are called anyons. In general case of $n$-particle state $\psi\left(\mathbf{r}_{1}, \ldots, \mathbf{r}_{n}\right)$, the topological classes of trajectories which take these particles from initial positions $\left(t_{i}, \mathbf{r}_{1}, \ldots, \mathbf{r}_{n}\right)$ to final position $\left(t_{f}, \mathbf{r}_{1}, \ldots ., \mathbf{r}_{n}\right)$ is in one-to-one correspondence with the elements of the braid group $B_{n}$. In figure 1 is a description of anyon worldlines originating from a plane with a characteristic fundamental braid.

Braid is an everyday concept but the formal definition of an $n$-braid as a topological object was given by Artin[10]. Consider two parallel frames with each frame as a plane in euclidean 3-space. Let $\mathbf{p}_{i}(i=1, \ldots, n)$ be distinct mark ponts on the lower frame $\Sigma_{L}$ and denote their orthogonal projections onto the upper frame $\Sigma_{U}$ as $\mathbf{q}_{i}$. Each $\mathbf{p}_{i}$ can be joined with $\mathbf{q}_{j}$ by means of strings which intersect any parrallel plane between $\Sigma_{L}$ and $\Sigma_{U}$ exactly once. 
A braid is an intertwining of some number of strings $f_{i}: I=[0,1] \longmapsto \Sigma$ such that:

1. $f_{i}(0)=\mathbf{p}_{i}$,

2. $f_{i}(1)=\mathbf{p}_{\pi(i)}=\mathbf{q}_{i}$ for some for some permutation $\pi$,

3. Given that $i \neq j$, then $f_{i}(t) \neq f_{j}(t)$ for each $t \in I$.

The braid group $B_{n}$ therefore consists of the set of all braids with $n$ strings under multiplication operation given by glueing. The braid group is furnished in a presentation provided in the following theorem:

Theorem $(\operatorname{Artin}[10])$ : Let $\mathcal{B}_{n}$ be a group generated by $\sigma_{1}, \sigma_{2}, \ldots, \sigma_{n-1}$ modulo the following relations:

1. $\sigma_{i} \sigma_{j}=\sigma_{j} \sigma_{i}$ for $|i-j| \geq 2$.

2. $\sigma_{i} \sigma_{i+1} \sigma_{i}=\sigma_{i+1} \sigma_{i} \sigma_{i+1}$ for $i=1, \ldots, n-2$,

There is an isomorphism $\rho: \mathcal{B}_{n} \longmapsto B_{n}$ determined by $\sigma_{i}$.

The elementary braids are those generated by $\sigma_{i}$ with just one crossing. The group $\mathcal{B}_{n}$ is different from permutation group in that $\sigma_{i}^{2} \neq 1$. As a consequence, while the permutation group is finite with $\left|S_{N}\right|=N$ !, the braid group is infinite. The two relations in the Artin's theorem are better revealed in figure 3 which notes that one composition can be smoothly deformed into the other. The first relation implies that the order of composition is important because the braid group is nonAbelian, e.g. $\sigma_{1} \sigma_{2} \neq \sigma_{2} \sigma_{1}$. The second relation is nothing but Yang-Baxter equation characterizing quantum integrable system. The Yang-Baxter equation is a statement that the two possible paths that three strings can take are different but equivalent.


$\sigma_{i} \sigma_{j}=\sigma_{j} \sigma_{i}$



$\sigma_{i+1} \sigma_{i} \sigma_{i+1}=\sigma_{i} \sigma_{i+1} \sigma_{i}$

Figure 3. Relations in Artin's braiding. 


\section{Chern-Simons Theory}

We have recognized Quantum field theory as a framework of model description of elementary particles. As we shall now demonstrate, the low energy behaviour of the fractional quantum Hall effect can be described by a certain planar Yang-Mill theory in the presence of Chern-Simons(CS) term [12]. In the following, we shall use both classical and quantum mechanical understanding to demonstrate that the source of Abelian and non-Abelian Chern-Simons theories have relevant anyonic features.

\subsection{Classical CS Invariant}

Let $\Sigma$ be a surface embedded in 3D Euclidean space with a compact boundary $\partial \Sigma$. The Riemannian metric determines a measure $d \mu_{\Sigma}$ on $\Sigma$ and defines the curvature of $\Sigma$ as a function $K: \Sigma \rightarrow \mathbb{R}$. The curvature is intrinsic in that it depends only on the induced metric on $\Sigma$, and not on the embedding of $\Sigma$ into space. The generalized Gauss-Bonnet formula is

$$
\int_{\Sigma} K d \mu_{\Sigma}+\int_{\partial \Sigma} \kappa_{\partial \Sigma} d \mu_{\partial \Sigma}=2 \pi \chi(\Sigma)
$$

where $\chi(\Sigma)$ is the Euler characteristic of $\Sigma$ and $\kappa$ is the total geodesic curvature of the boundary. The classical CS invariant[13] is a generalization of the total geodesic curvature.

Let $G$ be a finite Lie group and $t$ be its Lie algebra. We fix a certain closed oriented 3-manifold $\mathcal{M}$ with $m$-form $\Omega_{\mathcal{M}}^{m}(t)$ living on it. A connection $\Theta=\Theta_{\mu}^{a} t_{a} d x^{\mu}$ on the $G$-bundle over $\mathcal{M}$ is a skew-Hermitian matrix of 1-forms having trace zero. The curvature of $\Theta$ is a $t$-valued 2 -form $\Omega^{2}(\Theta)=d \Theta+\frac{1}{2}[\Theta \wedge \Theta]$ satisfying the Bianchi identity

$$
d \Omega+[\Theta \wedge \Omega]=0
$$

The Chern-Simons 3-form is given as

$$
\Omega^{3}(\Theta)=\Theta \wedge d \Theta+\frac{2}{3}[\Theta \wedge \Theta \wedge \Theta],
$$

and the corresponding classical CS invariant is

$$
S_{C S}(\Theta)=\frac{k}{4 \pi} \int_{\mathcal{M}} \operatorname{Tr}\left(\Theta \wedge d \Theta+\frac{2}{3}[\Theta \wedge \Theta \wedge \Theta]\right),
$$

with $k$ being the level of the theory. CS field theories exist for all odd and higher dimensions. The generalized CS form in $2 n+1$ dimensions reads as

$$
\Omega^{2 n+1}(\Theta)=\Theta \wedge(d \Theta)^{n}+\alpha_{1} \Theta^{3} \wedge(d \Theta)^{n-1}+\ldots . .+\alpha_{n} \Theta^{2 n+1}
$$

and $\alpha_{i} \in \mathbb{Q}$ is fixed.

\subsection{CS Model of Anyons}

Let $a_{\mu}$ be an emergent gauge fields of a certain Yang-Mill theory. In QFT, all physical information are encoded in the $n$-point correlation functions which can be extracted from functional differential of the partition function

$$
Z\left[a_{\mu}\right]=\int \mathcal{D} \Psi e^{i S\left[\Psi, a_{\mu}\right]}=e^{i S_{e f f}\left[a_{\mu}\right]},
$$


where $\Psi$ represent all dynamical fields which are integrated out towards arriving at the effective field action $S_{\text {eff }}$. The effective action describes some physics, accurate at the very low energy. Our first goal will be to identify the Chern-Simons action which describes the relevant physics of interest.

Abelian Chern-Simons theory emerge as a possible generalization of $3+1$ electromagnetic $U(1)$ gauge theory to $2+1$ dimensions. Locally in four spacetime dimensions, gauge connection $a_{\mu}$ is described by massless degrees of freedom, whose dynamics are encoded in the Maxwell's equations obtained by extremizing the action

$$
S_{\text {maxwell }}\left[a_{\mu}\right]=-\frac{1}{4 e^{2}} \int_{\mathcal{M}} d^{4} x f_{\mu \nu} f^{\mu \nu}
$$

where $f_{\mu \nu}=\partial_{\mu} a_{\nu}-\partial_{\nu} a_{\mu}$ is the curvature known as the electromagnetic field strength. We adopt a manifold $\mathcal{M}$ as a space on which the theory is formulated. We assume $\mathcal{M}$ has trivial topology with boundary $\partial \mathcal{M}$ arbitrarily far from particle worldlines, so that all field vanish on the boundary. The action is the most general Lorentz invariant action compatible with gauge transformations $a_{\mu} \rightarrow a_{\mu}+\partial_{\mu} \alpha$. The massless gauge field is in turn a consequence of gauge invariance which forbids some forms of interaction including a mass term. However in three spacetime dimensions, new interaction terms is allowed in the full action

$$
S=S_{\text {maxwell }}+S_{C S}
$$

which can potentially change the dynamics of the system. This term establishes the Abelian Chern-Simons action

$$
S_{C S}[a]=\frac{k}{4 \pi} \int_{\mathcal{M}} d^{3} x \epsilon^{\mu \nu \rho} a_{\mu} \partial_{\nu} a_{\rho}+(\ldots \ldots)
$$

where (......) may include some higher derivative terms. Before we explain why this action encodes an effective field theory describing the quantum hall system, it might be useful to generalize the Chern-Simons action into non-Abelian just as in the usual QFT formalism. We recognize the relevant Lie gauge group $G=S U(N)$ with $a_{\mu}=a_{\mu}^{c} t^{c}$ considered as $\operatorname{Lie}(G)$-valued gauge potential. The killing form $\kappa^{a b}=\operatorname{tr}\left(t^{a} t^{b}\right)=\frac{1}{2} \delta^{a b}$ can be used to raise and lower indices of the Lie-algebra. The curvature associated with the connection is

$$
f_{\mu \nu}=\partial_{\mu} a_{\nu}-\partial_{\nu} a_{\mu}-i\left[a_{\mu}, a_{\nu}\right]
$$

The full action in $2+1$ dimension is therefore nothing but a quantum Yang-Mills theory, with an action consisting of the Chern-Simons term that has additional 3-point gauge structure, i.e.

$$
S=S_{Y M}+S_{C S}
$$

with

$$
S_{C S}[a]=\frac{k}{4 \pi} \int_{\mathcal{M}} d^{3} x \epsilon^{\mu \nu \rho} \operatorname{Tr}\left(a_{\mu} \partial_{\nu} a_{\rho}-\frac{2 i}{3} a_{\mu} a_{\nu} a_{\rho}\right) .
$$

This is the action of level $k$ non-Abelian Chern-Simons theory with gauge group $G_{k}$. It takes the same structure as the Chern-Simons invariant (3.3). The most important gauge group for the purpose of our study will be $S U(2)_{k}$.

So, what are the features of Chern-simons theory that makes it a good effective field theory of the quantum hall system? In the following, we shall highlight both the features and some physics associated with the hall system. 
- The Chern-Simons action has no metric notion, instead, the Levi-Civita $\epsilon^{\mu \nu \rho}$ plays the role of raising and lowering. As such, the CS action is invariant under all diffeomorphism. The consequence of this is that, all $n$-point correlation functions are independent of the metric of spacetime, i.e. $\frac{\delta}{\delta g_{\mu \nu}}\left\langle\mathcal{O}_{1}\left(x_{1}\right) \ldots . \mathcal{O}_{n}\left(x_{n}\right)\right\rangle=0$. This make the correlation functions topological invariants and Chern-Simons theory a good example of topological quantum field theory.

- On dimensional ground, the Chern-Simons term dominates over the Yang-Mill and (......) term in $2+1$ dimension as it contains only one derivative relative to higher derivatives in the other terms. With Yang-Mill and other terms suppressed, the relevant long distance physics of the hall system is completely described by the Chern-Simons effective local Lagrangian, provided that $k \neq 0$.

- The Chern-simons action is invariant under rotation but violate parity $(x \rightarrow-x)$ and time reversal $(t \rightarrow-t)$ invariance. This is exactly the feature exhibited by quantum hall system where parity and time reversal are broken due to the external or background magnetic field as particles are restricted to a plane.

- Conservation of current plus the fact that vector theory demands the gauge field couples with the current implies that the current is the curl of the vector potential. Extremizing the CS action (3.7), we have the current as

$$
J^{\mu}=\frac{\delta S_{C S}[a]}{\delta a_{\mu}}=\frac{k}{2 \pi} \epsilon^{\mu \nu \rho} \partial_{\nu} a_{\rho} \Longrightarrow J_{i}=-\frac{k}{2 \pi} \epsilon_{i j} E^{j} .
$$

This means that, Chern-Simons action describes a system with conductivity

$$
\sigma_{x y}=\frac{k}{2 \pi} .
$$

We shall soon understand that, indeed, this is the hall conductivity of the very quantum hall effect if we identify the Chern-Simons level $k$ with $\nu$ landau level of the quantum hall system.

- Under a gauge transformation

$$
a_{\mu} \longrightarrow a_{\mu}^{u}=u^{-1} a_{\mu} u+i u^{-1} \partial_{\mu} u
$$

with $u(x) \in G$, the Chern-Simons action (3.8) transforms as

$$
\begin{aligned}
S_{C S}[a] \longrightarrow S_{C S}\left[a^{u}\right]= & S_{C S}[a]+\frac{k}{4 \pi} \int_{\mathcal{M}} d^{3} x \epsilon^{\mu \nu \rho} \partial_{\mu} \operatorname{Tr}\left(\partial_{\nu} u u^{-1} a_{\rho}\right) \\
& +\frac{k}{12 \pi} \int_{\mathcal{M}} d^{3} x \epsilon^{\mu \nu \rho} \operatorname{Tr}\left(u^{-1} \partial_{\mu} u u^{-1} \partial_{\nu} u u^{-1} \partial_{\rho} u\right) .
\end{aligned}
$$

This means that the action changes by a total derivative and an additional term

$$
\mathbb{Z} \ni \varsigma(u)=\frac{1}{24 \pi^{2}} \int_{\mathcal{M}} d^{3} x \epsilon^{\mu \nu \rho} \operatorname{Tr}\left(u^{-1} \partial_{\mu} u u^{-1} \partial_{\nu} u u^{-1} \partial_{\rho} u\right)
$$

Recognizing the total derivative in (3.11), the purely surface integral vanishes on the boundary. This follows because we have demanded all field to vanish on the boundary. However, the term involving $\varsigma(u)$ does not necessarily vanish if we demand $u(x) \rightarrow \mathbb{1}$ at infinity over the entire argument. Since the gauge transformation $u(x)$ is defined throughout $\mathbb{R}^{3}$ and its argument is equivalent to $\mathbb{S}^{3}$, the local gauge transformation is a map induced by a change 
of coordinates, i.e. $u: \mathbb{S}^{3} \rightarrow G$, and $\varsigma(u)$ measures the number of time the mapping winds around the spacetime. This therefore implies that the Chern-Simons action transformaming as

$$
S_{C S}[a] \longrightarrow S_{C S}\left[a^{u}\right]=S_{C S}[a]+2 \pi k \varsigma(u)
$$

is not invariant under gauge transformation. At this point, it appears gauge invariance is an obstacle towards having CS as a good model description of anyon, however, this violation is precisely all that is left to produce exact same scenario of the hall conductivity. In QFT, the object that must actually demand full compliance of gauge invariance isn't the action itself, but the very partition function (3.5) that encodes all information of the theory. Indeed, if we demand the Chern-Simons level $k$ to be an integer, so that on reinstating the natural units $e=1=\hbar$, we can achieve

$$
\frac{\hbar k}{e^{2}} \in \mathbb{Z}
$$

then the partition function $Z\left[a_{\mu}\right]$ is always gauge invariant. In fact, by associating $\frac{\hbar k}{e^{2}}$ with the filling fraction $\nu$ in (3.9) i.e. $\nu=\frac{\hbar k}{e^{2}}$, we precisely reproduce the quantized hall conductivity $\sigma_{x y}=\frac{e^{2}}{2 \pi \hbar} \nu$ in (1.3). This is an important result arising from CS theory being quasi-invariant.

- It is worth noting that the hierarchy state filling fraction in (1.4) can be constructed from this formalism by introducing $N$ emergent gauge fields $a_{\mu}^{i}$ with $i=1, \ldots, N$. The full effective theory with quasiparticle excitations is given by[14]

$$
\mathcal{L}=\frac{1}{4 \pi} K_{i j} \epsilon^{\mu \nu \rho} a_{\mu}^{i} \partial_{\nu} a_{\rho}^{j}+\frac{1}{2 \pi} t_{i} \epsilon^{\mu \nu \rho} A_{\mu} \partial_{\nu} a_{\rho}^{i}
$$

where the theory is completely specified by the CS couplings $K_{i j}$ and the charge vector $t_{i}$. The $K$-matrix associated with the hierarchy in (1.4) is given as

$$
K_{i j}=\left[\begin{array}{cccc}
m & -1 & 0 & \ldots \\
-1 & \widetilde{m}_{1} & -1 & \\
0 & -1 & \widetilde{m}_{2} & \\
\vdots & & & \ddots
\end{array}\right]
$$

- A quick check into the field equation of the full theory shows that the gauge fields have acquired mass which decay exponentially according as $e^{-m r}$, thereby rendering them as short ranged. This shows that, just like in the Aharonov-Bohm effect, the field can take large values away from the sources.

By now, we have understood how Chern-Simons theory makes a good model description of certain quasiparticles similar to those arising from the quantum hall system. Our next goal is to compute the expectation values of CS observables and then study how it features the anyonic properties of the source. 


\section{Wilson Loops and Anyonic Statistics}

Solving a QFT is up to determination of the $n$-point correlation functions. For Chern-Simons theory, we would like to evaluate the topological invariants of the theory. These are essentially the expectation value of a certain observables $\mathcal{O}_{i}$ on the manifold $\mathcal{M}$, i.e.

$$
\left\langle\mathcal{O}_{1}\left(x_{1}\right) \ldots \mathcal{O}_{n}\left(x_{n}\right)\right\rangle=\frac{1}{Z} \int_{\mathcal{M}} \mathcal{D} a e^{i S_{C S}[a]} \mathcal{O}_{1}\left(x_{1}\right) \ldots \mathcal{O}_{n}\left(x_{n}\right) .
$$

In this evaluation, anyonic statistics will depends on the evolution of wavefunction under the braiding operation of worldlines of anyons. We already saw braiding as a topological property of anyons. We will now study how this arise from CS theoretic description.

\subsection{Abelian and non-Abelian}

Our first goal is to identify an appropriate observable on the manifold. Define the Chern-Simons source as a set of particles on worldline in $2+1$ spacetime dimensions. We would like to find a physical description for space of trajectory of charged particles living inside a Manifold $\mathcal{M}$ with boundary $\partial \mathcal{M}$ far away from worldlines, so that fields vanish on the boundary. Lets assign particle a spin $j$ in the representation of CS gauge group $G_{k}$. We demand that any appropriate observable respect gauge invariance as the symmetry of the theory. There is a certain set of gauge invariant functional integral that satisfy this purpose. It is called the Wilson loop.

Starting with the Abelian. Given a 1-form $a_{\mu}$ as the gauge connection, an important concept is holonomy $H_{C, j}$, defined as the parallel transport along a closed curve C:

$$
H_{C, j}[a]=\mathcal{P} \exp \left(i q \oint_{C} a_{\mu} d x^{\mu}\right)
$$

where $\mathcal{P}$ is the path-ordering operator and $q$ is the charge associated with the particle source with label $j$. Holonomies are important concept because any gauge invariant quantity involving the connection $a_{\mu}$ is a traces of holonomies. This gauge invariant object is the Abelian Wilson loop

$$
W_{C, j}[a]=\operatorname{Tr}\left(\mathcal{P} \exp i q \oint_{C} a_{\mu} d x^{\mu}\right) .
$$

A simple interpretation will be to consider a source of quasiparticle-quasihole pair of type $j$, created from the vacuum, evolves along Wilson loop and then fused back to the vacuum. This process is described in figure 4. We shall soon see that the Chern-Simons level $k$ restricts the allowed set of spin representation $j$ propagating in the loop.

Loosely speaking, a collection of disjoint loops $\left(C_{i}, i=1, \ldots N\right)$ is called link $(\mathrm{L})$, so that we can define the product of Wilson loops as

$$
W_{L, R}[a]=\operatorname{Tr}\left(\mathcal{P} \prod_{i=1}^{N} \exp i q_{i} \oint_{C_{i}} a_{\mu} d x^{\mu}\right) .
$$

We can generalize the Wilson loop into non-Abelian by recognising that the gauge connection takes its value from the Lie algebra spanned by $t^{c}$. We can set the charge as $q=2 j$ and define $t_{(j)}^{c}=2 j t^{c}$. The the non-Abelian Wilson loops in the representation $\mathrm{R}$ of the gauge group $G_{k}$ is defined as

$$
W_{L, R}[a]=\operatorname{Tr}_{R}\left(\mathcal{P} \prod_{i=1}^{N} \exp i \oint_{C_{i}} t_{(j)}^{c} a_{\mu}^{c} d x^{\mu}\right) .
$$






Figure 4. Worldlines of particles in a 3D Euclidean space $\mathcal{M}$. Wilson lines are defined by loops $C_{1}$ and $C_{2}$. The two loops together form a link. The link intersect disk $\Sigma_{0}$ at points $P_{i}$. The diagram depicts four particle sources pairwisely created from the vacuum; the source braided and then fused back to the vacuum

\subsection{Expectation Value}

\section{Abelian CS}

As we have noted in (4.1), the expectation value of the Wilson loop is given as

$$
\left\langle W_{L, j}\right\rangle=\frac{\int_{\mathcal{M}} \mathcal{D} a W_{L, j} e^{i S_{C S}[a]}}{\int_{\mathcal{M}} \mathcal{D} a e^{i S_{C S}[a]}} .
$$

Consider an Abelian Chern-Simons theory (3.7) and a link of disjoint loops $C_{i}$ associated with charges $q_{i}$ living in the worldvolume $\mathcal{M}$. Figure 4 depicts the case of two loops link. We are left with a process in a link configuration such that, particles are created pairwisely from the vacuum, propagate through the worldlines $C_{i}$ and then fuse back to the vacuum. It is not hard to show in the case of Abelian CS, that the expectation value of the Wilson loops is given by[15]

$$
\left\langle W_{L, j}\right\rangle=\exp \left(\frac{i 2 \pi}{k} \sum_{i, j} q_{i} q_{j} \Phi\left(C_{i}, C_{j}\right)\right),
$$

with Gauss linking number

$$
\mathbb{Z} \ni \Phi\left(C_{i}, C_{j}\right)=\frac{1}{4 \pi} \oint_{C_{i}} d x^{\mu} \oint_{C_{j}} d y^{\nu} \epsilon^{\mu \nu \rho} \frac{(x-y)^{\rho}}{|x-y|^{3}}, \text { for } i \neq j .
$$

$\Phi\left(C_{i}, C_{j}\right)$ and $\left\langle W_{L, j}\right\rangle$ are topological invariants ( of link. $\Phi\left(C_{i}, C_{j}\right)$ measures the number of times one loop $C_{i}$ winds around another loop $C_{j}$. It may be positive or negative depending on the orientation of the loops involved.

\section{Comments:}

1. The Gauss linking number is divergent for $i=j$. This can be resolved by a framing prescription that displace one of the loops, say $C_{i}$, slightly in a given direction to a loop $C_{i}^{\prime}$. As such, a ribbon structure with boundaries $C_{i}$ and $C_{i}^{\prime}$ is formed. This results in a well defined Gauss 
linking number.

2. To display the anyonic nature of the source, we consider the trivial loops $L_{0}$ corresponding to disentangled loops. We note that an entagled loop corresponds to a braided sources characterizing anyons. Consider two loops $C_{1}$ and $C_{2}$ winding one another once according as $\Phi\left(C_{1}, C_{2}\right)= \pm 1$ so that their trivial link corresponds to two disentangled loops. Then braiding operation according to the expectation value (4.7) is given as

$$
\left\langle W_{L, j}\right\rangle=\exp \left(i \frac{4 q^{2}}{k}\right)\left\langle W_{L_{0}, j}\right\rangle \text {. }
$$

Indeed, the braiding operation left a phase. This implies that the Chern-Simons sources have anyonic statistics.

3. The generalization of the expectation value to non-Abelian CS theory is complicated by large class of links having the same Gauss linking number $\Phi\left(C_{i}, C_{j}\right)$. We need a different algorithm to resolve this situation. This algorithm aim to evaluate a certain knot invariant called the Jones polynomials. However for now, consider two sources of a non-Abelian $S U(2)_{k}$ CS gauge group characterized by a label $j$. Fusion of $j_{1}$ and $j_{2}$ is dictated by vector composition

$$
j_{1} \otimes j_{2}=\sum_{\left|j_{1}-j_{2}\right|}^{\lambda\left(j_{1}, j_{2}\right)} j .
$$

Are all $j$ allowed by fusion rules in CS theory? The answer is no as not all $j$ gives distinct source. Indeed, expectation value of the non-Abelian Wilson loops for $j_{\max }>\frac{k}{2}$ produce the same result as those of the representation with $0 \leq j \leq \frac{k}{2}$. Therefore, there are only $k+1$ allowed representations: $0, \frac{1}{2}, \ldots, \frac{k}{2}-$ with

$$
\lambda\left(j_{1}, j_{2}\right)= \begin{cases}j_{1}+j_{2}, & j_{1}+j_{2} \leq \frac{k}{2} \\ k-j_{1}-j_{2}, & j_{1}+j_{2}>\frac{k}{2}\end{cases}
$$

\section{Non-Abelian CS}

We noted earlier that the generalization of the expectation value to non-Abelian CS theory is not straightforward. The complication arises due to a large class of links having the same Gauss linking number $\Phi\left(C_{i}, C_{j}\right)$. We state forward at this point that, we are yet to have enough technique to confront the problem. However, we shall lay down the formal approach towards the computation of the partition function. Here, we shall sketch out Witten's ideas. This idea will be used later to resolve through the problem at hand.

\section{Witten's Procedure}

Consider the three manifold $M$ (picture it as $S^{3}$ ). Inside this manifold is our theory, i.e. Wilson loop $W_{C, j}$ is living therein as shown in figure 5 .

- Cut the 3-manifold $\mathcal{M}$ along along a Riemann surface $\Sigma$, into two 3-manifolds $\mathcal{M}_{1}$ and $\mathcal{M}_{2}$. Obviously, $\mathcal{M}_{1}$ and $\mathcal{M}_{2}$ contain boundary surfaces, say $\Sigma_{1}$ and $\Sigma_{2}$, so that after cutting, the boundary of the pieces become $\partial \mathcal{M}_{1}=\Sigma_{1} \cup \Sigma$ and $\partial \mathcal{M}_{2}=\Sigma_{2} \cup \Sigma^{*}$. $\Sigma^{*}$ is essentially $\Sigma$ with opposite orientation. The surface of the cut would appear like $\Sigma \times \mathbb{R}$, where $\Sigma$ is the spatial part and $\mathbb{R}$ is the time direction in a similar way to figure 4 . 



c


a $\mathrm{M}$


Figure 5. Witten's Quantization procedure. Left: (a) depicts the cutting procedure of a general manifold $\mathcal{M}$ into $\mathcal{M}_{1}$ and $\mathcal{M}_{2}$. One can do similar cutting procedure for a three sphere $S^{3}$ in (b). Right: (a) shows the inclusion of Wilson loop $W$ and the cutting of the three manifold $\mathcal{M}$. Wilson loop carrying representation $R$ of the gauge group pierce through Riemann surface $\Sigma$ and leaves marked points on it. (b) shows that, near the cut, $\mathcal{M}$ looks locally like $\Sigma \times \mathbb{R}$.

- Canonically quantize CS theory on $\Sigma$ and construct the physical Hilbert space $\mathcal{H}_{\Sigma}$. A path integral over $\mathcal{M}_{1}$ is a quantum state $\left|\psi_{\mathcal{M}_{1}}\right\rangle \in \mathcal{H}_{\Sigma}$. Since the surface $\Sigma^{*}$ of $\partial \mathcal{M}_{2}$ has opposite orientation of $\Sigma$, the physical Hilbert space on $\partial \mathcal{M}_{2}$ is just a dual space $\mathcal{H}_{\Sigma}^{*}$ and the path integral over it is denoted as $\left\langle\psi_{\mathcal{M}_{2}}\right| \in \mathcal{H}_{\Sigma}^{*}$.

- Heegaard splitting and Gluing: Let Every $\mathcal{M}, \mathcal{M}_{1}$, and $\mathcal{M}_{2}$ be compact, closed, connected, orientable 3-manifolds and $\partial \mathcal{M}_{1}=\partial \mathcal{M}_{2}=\Sigma=\mathcal{M}_{1} \cap \mathcal{M}_{2}$ be a Riemann surface. $\mathcal{M}$ admits Heegaard splitting $\left(\Sigma, \mathcal{M}_{1}, \mathcal{M}_{2}\right)$ such that, given an orientation reversing homeomorphism $f: \Sigma \rightarrow \Sigma$, there is a gluing procedure leading to $\mathcal{M}=\mathcal{M}_{1} \cup_{f} \mathcal{M}_{2}$ [17].

- Atiyah's Axioms: A TQFT is a functor $\mathcal{Z}: n C o b \rightarrow \mathcal{H}$ with respect to orientation preserving diffeomorphisms of $\Sigma$ and $\mathcal{M}$. This statement basically means that, we assign a Hilbert spaces $\mathcal{H}_{\Sigma}$ and $\mathcal{H}_{\mathcal{M}}$ to Rieman surface $\Sigma$ and 3-manifolds $\mathcal{M}$ respectively. Functor $\mathcal{Z}$ is involutory, i.e. $\mathcal{Z}\left(\Sigma^{*}\right)=\mathcal{Z}(\Sigma)^{*}$ and multiplicative, i.e. $\mathcal{Z}\left(\Sigma_{1} \cup \Sigma_{2}\right)=\mathcal{Z}\left(\Sigma_{1}\right) \otimes \mathcal{Z}\left(\Sigma_{2}\right)$ [18].

- The homeomorphism $f: \Sigma \rightarrow \Sigma$ induces a homeomorphism $U_{f}: \mathcal{H}_{\Sigma} \rightarrow \mathcal{H}_{\Sigma}^{*}$. $U_{f}$ is a unitary operator so that we can compute the partition function $Z(\mathcal{M})=\left\langle\psi_{\mathcal{M}_{2}}\left|U_{f}\right| \psi_{\mathcal{M}_{1}}\right\rangle$ on $\mathcal{M}$.

- Now insert the Wilson loops in $\mathcal{M}$. On $\Sigma$ is left mark points or punctures $P_{i}$ with each assigned a representation $R_{i}$ of the gauge group $G_{k}$. This therefore allows us to quantize the theory on $\Sigma \times \mathbb{R}$, and the theory is solved on evaluating the expectation value

$$
\left\langle W_{C_{1}, R_{1}} \ldots W_{C_{n}, R_{n}}\right\rangle=\frac{1}{Z} \int_{\mathcal{M}} \mathcal{D} a e^{i S_{C S}[a]} W_{C_{1}, R_{1}} \ldots W_{C_{n}, R_{n}} .
$$




\section{The Jones Polynomial}

In this section, our goal is to get accustomed to the machinery we shall use in evaluating the expectation value of the non-Abelian Wilson loops. While the concept associated with the topic in this section is broad as an area of interest, all we need for our purpose is a mathematical concept called the Jones polynomial. Our knot excursion shall therefore be brief.

\subsection{Knots and Links}

Here, we start with a basic overview of knot theory leading to the Jones polynomial.

- A knot is an embedding of a closed curve in $\mathbb{R}^{3}$; a circle is a trivial knot. A link is a finite family of disjoint union of knots. This implies that a knot is a link with a single closed curve. It is worth mentioning that every link is the closure of some braid.

- Knots are 3D objects but mostly studied through knot diagram obtained by projecting the $3 \mathrm{D}$ object onto a plane $\mathbb{R}^{2}$ just as depicted in figure 6 . The non-trivial knots in figure 6 are examples showing that there is no unique way of embedding a closed curve in $\mathbb{R}^{3}$. Any closed curve can be knotted in different ways having different number of crossings. This leaves a question in knot theory: when are two knots, or perhaps two knot diagrams, equivalent?


Figure 6. The first two diagrams are non-trivial knots with 3 and 4 crossings respectively. The third diagram is the Hopf link.

- Existence of orientation preserving homeomorphism between two knot diagrams may provide equivalence of two knots. However, this is not always true and such homeomorphism is hard to find in practice.

- Two links are equivalent or isotopic if they differ by a finite sequence of local sequence of Reidemeister moves and an orientation- preserving homeomorphism of the plane. There are three types of Reidemeister moves as shown in figure 7. Any homeomorphism of the plane must preserve all crossing information.



Type I



Type II



Type III

Figure 7. Three types of Reidemeister moves 
- By orientation of a link, we means a choice of direction of trajectory around each component of the link. A cross change can be done in a diagram of an oriented link. Such crossing is a local modification of the type in figure 8. They are completely different from Reidemeister moves.



Figure 8. Two types of crossing change

- Any link can be unknotted by a finite number of cross changes. This cross changes allows for construction of link invariants. A link invariant assigns same object to links in the same isotopy class. An example of link invariant is the Jones polynomial.

Basically, the Jones polynomial is an invariant of link. Of all different model, Jones polynomial[16] is simpler to define in the light of Kauffman bracket polynomial. There are The Kauffman bracket is easier computed through skein relations.

Definition. It is a function from unoriented link diagrams to Laurent polynomials in variable A using the following rules:

1. $\langle\bigcirc\rangle=1, ; \quad \bigcirc=$ trivial link.

2. $\langle L \cup \bigcirc\rangle=\left(-A^{2}-A^{-2}\right)\langle L\rangle ; \quad L=$ link diagram.

3. \langle\rangle$\rangle=A\langle\bumpeq\rangle+A^{-1}\langle\rangle\langle\rangle$.

As an example, the computation of the bracket polynomial of Trefoil knot using skein relation is shown in figure 9. The bracket polynomial is invariant under type II and type III Reidemeister moves. Under type I move, the invariance is up to some pre-factors. This prefactor anomaly is as a result writhe.

Definition. The writhe $w(L)$ of a diagram $L$ of an oriented link is the sum of the signs of the crossing change defined according as the convention in figure 8.

$w(L)$ is invariant under a type II or III Reidemeister move in $L$, but it does change by +1 or -1 under type I Reidemeister move in $\mathrm{L}$. This is in fact the reason why bracket polynomial is not invariant due to some prefactors. It turns out that multiplication of bracket polynomial by $(-A)^{-3 w(L)}$ cancels out all the prefactors left by Type I Reidemeister moves. This leads to the definition that the polynomial

$$
X(L)=(-A)^{-3 w(L)}\langle L\rangle,
$$

is an invariant of the oriented link $L$. The Jones polynomials is a special case with variable $A=q^{-1 / 4}$. 


$$
\begin{aligned}
& \langle(D)\rangle=A\langle\bigcirc)\rangle+A^{-1}\langle(n)
\end{aligned}
$$



$$
\begin{aligned}
& \left.=A^{3}\langle 00\rangle+A\langle 0)\right\rangle+A\left\langle(0)+A^{-1}\langle(0)\rangle\right.
\end{aligned}
$$

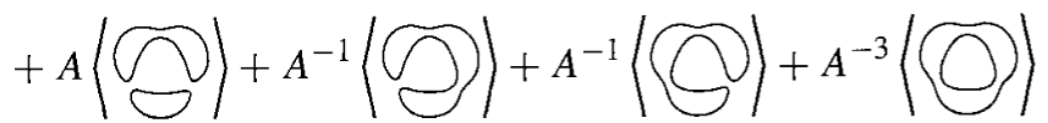

$$
\begin{aligned}
& =A^{3}\left(-A^{2}-A^{-2}\right)^{2}+A\left(-A^{2}-A^{-2}\right)+A\left(-A^{2}-A^{-2}\right)+A^{-1} \\
& +A\left(-A^{2}-A^{-2}\right)+A^{-1}+A^{-1}+A^{-3}\left(-A^{2}-A^{-2}\right) \\
& =A^{7}-A^{3}-A^{-5} \text {. }
\end{aligned}
$$

Figure 9. Bracket polynomial of Trefoil knot

Definition. The Jones polynomial $V(L)$ of an oriented link $L$ is a Laurent polynomial in variable $q^{1 / 2}$ assigned to an oriented link $L$ defined by

$$
V(L)=(-A)^{-3 w(L)}\langle L\rangle_{A^{-2}=q^{1 / 2}}
$$

satisfying the following properties:

1. $V($ trivial knot $)=1$

2. if $L$ is isotopic to $L^{\prime}$, then $V(L)=V\left(L^{\prime}\right)$; the converse is not necessarily true.

3. $q^{-1} V\left(L_{+}\right)-q V\left(L_{-}\right)+\left(q^{-1 / 2}-q^{1 / 2}\right) V\left(L_{0}\right)=0$, where $L_{0}, L_{-}$and $L_{+}$are Skein relation in Figure 10 showing identical oriented link diagrams but differ only at one crossing.


Figure 10. Skein relation

The Jones polynomial was discovered through a new representation of the Artin braid group. It has proved very useful in that, it has strong feature of distinguishing one link from another. This includes mirror images of link, which can be obtained for the Jones polynomial by replacing $q$ with $q^{-1}$. Thus far, we have learn that any link can be unknotted by a finite number of crossing and that, the Jones polynomial allows us to evaluate the link invariant from the skein diagram. 


\section{Non-Abelian CS theory}

Now, we shall return to Physics and embark on studying the Witten's approach to solving nonAbelian Chern-Simons theory. At the concluding part of section 4, we already highlighted the the basic procedure for this section. We shall now discuss them step-by-step. The first non-trivial step is the Quantization of CS theory on $\Sigma \times \mathbb{R}$. As we shall realize, the canonical approach for evaluating the Wilson loops will be based on constructing the Hilbert space on a Riemann surface $\Sigma$ with punctures.

\subsection{Quantization on $\Sigma \times \mathbb{R}$}

We return back to the non-Abelian CS theory (3.8). In the euclidean theory, we can well write the Lagrangian density as

$$
\mathcal{L}=\frac{k}{8 \pi} \int_{\mathcal{M}} \epsilon^{i j k} \operatorname{Tr}\left(a_{i}\left(\partial_{j} a_{k}-\partial_{k} a_{j}\right)+\frac{2}{3} a_{i}\left[a_{j}, a_{k}\right]\right)
$$

For now, we shall ignore the Wilson loop, and canonically quantize CS theory. Let us denotes the connection in the $\Sigma$-direction as $a_{i=1,2}$ and $a_{0}$ as the gauge connection in the $\mathbb{R}$-direction. Towards quantization, we recognize that there exist a natural gauge $a_{0}=0$ on $\Sigma \times \mathbb{R}$. With this gauge, the nonlinearity is tractable and the Lagrangian density reads

$$
\mathcal{L}=\frac{k}{4 \pi} \int_{\mathbb{R}} d t \int_{\Sigma} \epsilon^{i j} \operatorname{Tr}\left(a_{i} \frac{\partial}{\partial t} a_{j}+a_{0} f_{i j}\right)
$$

In this way, $a_{i}$ is a canonical conjugate of $\frac{k}{4 \pi} \epsilon^{i j} a_{j}$. This implies that, in CS theory without the Wilson loops, components of gauge connection are canonical conjugate to one another. Furthermore, we recognize that $a_{0}$ is a Lagrangian multiplier that enforces a Gauss constraint $f_{i j}=0$; as such, we are left with the quantization of contained system. Constrained systems can be quantized in two different but equivalent ways, namely,

- first canonically quantize and then enforces the Gauss constraint at the quantum level. In this way, we have the commutation relation as

$$
\left[a_{i}^{b}(x), a_{j}^{c}(y)\right]=\frac{2 \pi i}{k} \epsilon_{i j} \delta^{b c} \delta^{2}(x-y),
$$

subject to the Gauss constraint $\epsilon^{i j} f_{i j}^{a}=0$ on the wave solution.

- Impose the Gauss constraint $\epsilon^{i j} f_{i j}^{a}=0$ classically and then quantize the resulting degrees of freedom. So, we need first solve the constraint on $\Sigma$. This is equivalent to a problem of solving for the moduli space of flat connections on $\Sigma$ for which curvature vanishes. A connection is expected to detail a way to do parallel transport in a principal bundle over $\Sigma$ with structure group $G_{k}$. Therefore, for any closed loop in $\Sigma$, a connection determines a holonomy, i.e. the solutions of the constraint are parametrized by the holonomies of the gauge connection around a cycle in $\Sigma$. For a genus $g$ surface $\Sigma$, and a gauge group $G_{k}$, the dimension of the moduli space of flat connection is $(2 g-2) \operatorname{dim} G_{k}$.

- We are particularly interested in $\Sigma=S^{2}$. However, there are no flat connection on $S^{2}$, thereby rendering quantization trivial with with just a unique state. As such, $\operatorname{dim} \mathcal{H}_{\Sigma}=1$. 
- For a genus $g \geq 1$ surface $\Sigma_{g}$ and gauge group $S U(2)_{k}$, the dimension of the Hilbert space is given by

$$
\operatorname{dim} \mathcal{H}_{\Sigma_{g}}=\left(\frac{k+2}{2}\right)^{g-1} \sum_{j=0}^{k}\left(\sin \frac{(j+1) \pi}{k+2}\right)^{2-2 g}
$$

This is the Verlinde formula[19] arising as a consequence of the fact that, the modular $S$ Matrix implementing modular transformation $\tau \rightarrow-\frac{1}{\tau}$ diagonalizes the fusion rule. A very important feature of this formula is that, it is an integer and a finite polynomial in $k$.

\subsubsection{Quantization on $\Sigma$ with punctures}

So far, we have quantized the CS theory on $\Sigma$ without the Wilson loop. On inclusion of the Wilson loop, the key insight is to recognise that the worldlines of particles will leave markpoints (punctures) of static charges at points $\xi_{i}$ on the Riemann surface $\Sigma$ as the loops pierce through it. Each point $\xi_{i}$ corresponds to a representation $R_{i}$ of the gauge group $G_{k}$. Quantization of CS theory in the presence of Wilson loop is therefore up to finding the Hilbert space $\mathcal{H}_{\Sigma, \xi_{i}, R_{i}}$ associated to the surface $\Sigma$ with the choice of markpoint $\xi_{i}$ corresponding to $R_{i}$. As the Wilson lines correspond to static non-abelian charges, the Gauss constraint is therefore modified as

$$
\frac{k}{8 \pi} \epsilon^{i j} f_{i j}^{c}(x)=\sum_{n=1}^{p} \delta^{2}\left(x-\xi_{n}\right) t_{(n)}^{c},
$$

where $t_{(r)}^{c}, c=1, \ldots, \operatorname{dim} G$ are the generator of the gauge group associated with static external charges placed at $\xi_{r}$. Following either of the earlier quantization approach result into rather quantization issues resolved as follows:

- the large $k$ limit, i.e. $k \rightarrow \infty$ corresponds to the weak coupling limit of the CS theory, so that we can do calssical analysis.

- Dirac quantization criterion applies to each $f_{i j}^{c}$.

- for large $k$, Dirac quantisation cannot be applied alongside with non-trivial charge on the right hand side of the Gauss constraint.

- possible resolution is for all charges to sum up to zero. This is indeed the case as equal quantities of positive and negative charge is expected no matter how weak the coupling is. As such, the net charge is zero. In non-Abelian theory, this implies that all of the charges must be coupled with trivial representation of the gauge group.

- i.e. these are all trivial representation arising from the decomposition of $\bigotimes_{i=1}^{n} R_{i}$ in the large $k$ limit and it is restricted to this.

- In the light of this resolution, the physical Hilbert space on $\Sigma=S^{2}$ with three marked points corresponding to representations $R_{i}, R_{j}$ and $\bar{R}_{k}$ is spanned by the orthonormal states of all possible fusion channels $\mathcal{V}_{i j}^{k}$ whose dimension is $\sum_{k} \mathcal{N}_{i j}^{k}$.

In the spirit of quantization, there is a geometric construction of the Hilbert space according as the Borel-Weil-Bott theorem. They constructed the Hilbert space as the space of holomorphic sections of line bundle leading to a linear representation of $G$. In this formalism, all irreducible representation 
arise from such kind of construction, an understanding that become useful in quantization of ChernSirnons theory. The Hilbert space is given as

$$
\mathcal{H}_{\left(\Sigma ; \xi_{1}, R_{1} ; \ldots ; \xi_{n}, R_{n}\right)}=\bigotimes_{i=1}^{n} R_{i}^{G}=\left(R_{1} \otimes \ldots \otimes R_{n}\right)^{G} .
$$

- For $n=0$, there is only the trivial representation on $\Sigma$ and $\operatorname{dim} \mathcal{H}_{\Sigma}=1$.

- For $n=1$, the Hilbert space is $\mathcal{H}_{\Sigma ; \xi_{1}, R_{1}}=R_{1}^{G}$, so that $\mathcal{H}_{\Sigma ; \xi_{1}, R_{1}}=1$ if $\mathrm{R}$ is trivial and 0 otherwise.

- For $n=2, \operatorname{dim} \mathcal{H}_{\Sigma ; \xi_{1}, R_{1}, \xi_{2}, R_{2}}=\operatorname{dim}\left(R_{1} \otimes R_{2}\right)^{G}=1$ if $R_{2}$ is dual $R_{1}$ and 0 otherwise.

- For $n=3$, the $\operatorname{dim} \mathcal{H}_{\Sigma ; \xi_{i}, R_{i}, \xi_{j}, R_{j}, \xi_{k}, R_{k}}=\operatorname{dim}\left(R_{i} \otimes R_{j} \otimes R_{k}\right)^{G}=\mathcal{N}_{i j}^{k}$. The Hilbert space is spanned by the orthonormal states of all possible fusion channels.

- Let $R$ be the representation of the gauge group $G=S U(N)$. With two Wilson loops, there are four marked points, i.e. $n=4$, corresponding to representations $R, R, \bar{R}$ and $\bar{R}$. There are two distinct irreducible representations arising from decomposition $R \otimes R=E_{1} \oplus E_{2}$. $E_{1}$ is the symmetric representation generated by $\frac{1}{2}\left(e_{i} \otimes e_{j}+e_{j} \otimes e_{i}\right)$, and $E_{2}$ is the antisymmetric representation generated by $\frac{1}{2}\left(e_{i} \otimes e_{j}-e_{j} \otimes e_{i}\right)$, so that we have

$$
R \otimes R \otimes \bar{R} \otimes \bar{R}=\left(E_{1} \oplus E_{2}\right) \otimes\left(\bar{E}_{1} \oplus \bar{E}_{2}\right) .
$$

For $\mathrm{SU}(2)$, this implies that

$$
\operatorname{dim} \mathcal{H}_{\Sigma ; \xi_{1}, R_{1}, \xi_{2}, R_{2}, \xi_{3}, R_{3}, \xi_{4}, R_{4}}=\operatorname{dim}(R \otimes R \otimes \bar{R} \otimes \bar{R})^{G}=2,
$$

i.e. $S U(2)$ is generated by $E_{1} \otimes \bar{E}_{1}$ and there are only two possible fusion outcomes. This is the central idea we shall use to explain skein relations in Jones polynomials.

\subsection{Evaluation by TQFT}

Before we embark on computation, we first establish some factorization properties which has potential of reducing evaluation of many loops to that of a single loop. This will follow from our earlier observation depicted in figure 5 and the TQFT axioms that follows it.

- Factorization I: Let $\mathcal{M}_{1}$ and $\mathcal{M}_{2}$ be two three manifolds and $\mathcal{M}=\mathcal{M}_{1} \# \mathcal{M}_{2}$ be the connected sum (\#) of two $\mathcal{M}_{1}$ and $\mathcal{M}_{2}$ along $S^{2}$. Following Atiyah's axiom, we deduce a factorization

$$
Z(M) \cdot Z\left(S^{3}\right)=Z\left(\mathcal{M}_{1}\right) \cdot Z\left(\mathcal{M}_{1}\right)
$$

where $Z(X)$ denotes the partition function over any three manifold $X$ inside which knots may live. $S^{3}$ is the three sphere filling in $S^{2}$; it carries no knot and the partition function over it is $Z\left(S^{3}\right)$. (6.7) implies that

$$
\frac{Z(M)}{Z\left(S^{3}\right)}=\frac{Z\left(\mathcal{M}_{1}\right)}{Z\left(S^{3}\right)} \cdot \frac{Z\left(\mathcal{M}_{1}\right)}{Z\left(S^{3}\right)}
$$

- Factorization II: Given any link $L$, living in a three manifold $\mathcal{M}$, the expectation value of $L$ is given as

$$
\langle L\rangle=\frac{Z(M, L)}{Z(M)} .
$$


- Factorization III: Let $C_{1}, \ldots, C_{n}$ be $n$ unlinked, unknotted loops living on the three sphere $S^{3}$. Following (6.8) and (6.9), then

$$
\left\langle C_{1}, \ldots, C_{n}\right\rangle=\prod_{k=1}^{n}\left\langle C_{k}\right\rangle .
$$

\subsubsection{Unknotting Procedure}

We shall now present SU(2) Chern-Simons theory and its connection with the Skein relation. We shall not repeat the entire story associating with figure 5 again, rather, we remind ourselves that our goal is to evaluate the partition function $Z(L)$ of a link $L$ living on a three manifold $\mathcal{M}$, from which the expectation value $\left\langle W_{L}\right\rangle$ can be computed. Witten derived the rule for unknotting the link as follows:

- Embed a link $L$ in a general three manifold $\mathcal{M}$ and let the link components (i.e. each Wilson loop) carry fundamental representation $R$ of the $S U(2)$ gauge group. The link features a crossing configuration (see figure 11a). Imagine inserting a small sphere around the crossing configuration. The sphere would intersect the link at four marked points, so that it cuts $\mathcal{M}$ into two pieces corresponding to $\mathcal{M}_{L}$ and $\mathcal{M}_{R}$ in Figure 11b.


Figure 11. Cutting procedure of three manifold $\mathcal{M}$

- In this way, a part of the link $L$ inside $\mathcal{M}_{R}$ has a single crossing and the other part is contained in $\mathcal{M}_{L}$. However, $\mathcal{M}_{L}$ and $\mathcal{M}_{R}$ shares same boundary $\Sigma=S^{2}$.

- We already quantized the theory on the boundary $\Sigma=S^{2}$. We therefore associate physical Hilbert spaces $\mathcal{H}_{L}$ and $\mathcal{H}_{R}$ with the boundaries of $\mathcal{M}_{L}$ and $\mathcal{M}_{R}$ respectively with $\mathcal{H}_{L}=\mathcal{H}_{R}^{*}$. We know the Hilbert spaces are two dimensional according as (6.6). Feynman path integral over $\mathcal{M}_{L}$ and $\mathcal{M}_{R}$ yields vectors $\left\langle\psi_{\mathcal{M}_{L}}\right| \in \mathcal{H}_{L}$ and $\left|\psi_{\mathcal{M}_{R}}\right\rangle \in \mathcal{H}_{R}$ respectively.

- The partition function then gives $Z(L)=\left\langle\psi_{\mathcal{M}_{L}}\left|U_{f}\right| \psi_{\mathcal{M}_{R}}\right\rangle$, where $U_{f}: \mathcal{H}_{L} \rightarrow \mathcal{H}_{R}$ is a unitary operator induced by orientation preserving homeomorphism $f$ used in the gluing procedure. 
- Again, $\mathcal{H}_{L}$ and $\mathcal{H}_{R}$ are two dimensional. Since a linear combination of three vectors in 2D vector space vanishes, then given $\left|\chi_{\mathcal{M}_{R}}\right\rangle,\left|\varphi_{\mathcal{M}_{R}}\right\rangle \in \mathcal{H}_{R}$, there is a linear relation

$$
\alpha\left|\psi_{\mathcal{M}_{R}}\right\rangle+\beta\left|\chi_{\mathcal{M}_{R}}\right\rangle+\gamma\left|\varphi_{\mathcal{M}_{R}}\right\rangle=0,
$$

and as a consequence,

$$
\begin{gathered}
\alpha\left\langle\psi_{\mathcal{M}_{L}}\left|U_{f}\right| \psi_{\mathcal{M}_{R}}\right\rangle+\beta\left\langle\psi_{\mathcal{M}_{L}}\left|U_{f}\right| \chi_{\mathcal{M}_{R}}\right\rangle+\gamma\left\langle\psi_{\mathcal{M}_{L}}\left|U_{f}\right| \varphi_{\mathcal{M}_{R}}\right\rangle=0, \\
\alpha Z(L)+\beta Z\left(L^{\prime}\right)+\gamma Z\left(L^{\prime \prime}\right)=0
\end{gathered}
$$

where $L^{\prime}$ and $L^{\prime \prime}$ are links associated with respective three manifolds $\mathcal{M}^{\prime}$ and $\mathcal{M}^{\prime \prime}$. While we shall take $\mathcal{M}^{\prime}=\mathcal{M}=\mathcal{M}^{\prime \prime}$, both $L^{\prime}$ and $L^{\prime \prime}$ carry worldline braiding different from that of $L$ as a consequence of replacing three $\mathcal{M}_{R}$ with $\mathcal{M}_{R}^{\prime}$ and $\mathcal{M}_{R}^{\prime \prime}$ respectively and application of braiding rule.



Figure 12. Braiding operation of a section of Wilson link living in $\mathcal{M}_{R}$

- As shown in Figure 12, clockwise exchange of the first two marked points on the boundary of $\mathcal{M}_{R}^{\prime}$ yield the link configuration $L_{R}$, while the anticlockwise exchange yield the configuration $L_{R}^{\prime \prime}$. This clockwise and anticlockwise exchange are respectively implemented by braiding unitary operator $\mathcal{B}^{-1}$ and $\mathcal{B}$, so that state corresponding to $L_{R}$ and $L_{R}^{\prime \prime}$ are respectively given as

$$
\left\{\begin{aligned}
\left|\psi_{\mathcal{M}_{R}}\right\rangle & =\mathcal{B}^{-1}\left|\chi_{\mathcal{M}_{R}^{\prime}}\right\rangle=\mathcal{B}^{-1}\left|\chi_{\mathcal{M}_{R}}\right\rangle \\
\left|\varphi_{\mathcal{M}_{R}}\right\rangle & =\mathcal{B}\left|\chi_{\mathcal{M}_{R}^{\prime}}\right\rangle=\mathcal{B}\left|\chi_{\mathcal{M}_{R}}\right\rangle .
\end{aligned}\right.
$$

- (6.12) is further represented in Figure 13. Indeed, this diagram represents an expression related to the Skein relation encountered in section 5 as a property of the Jones polynomial.



Figure 13. Skein-Recursion relation for links 
- (6.12) is interpreted as follows. Considers three topologically equivalent closed contours corresponding to three links whose plane projections are identical outside a disc, and respectively have Skein relation inside the disc (see Figure 10 and 14). The expectation values of the three topologically equivalent closed contours weighted with $\alpha, \beta$ and $\gamma$ is zero, i.e. $\alpha Z(L)+\beta Z\left(L^{\prime}\right)+\gamma Z\left(L^{\prime \prime}\right)=0$ for three links $L, L^{\prime}$ and $L^{\prime \prime}$.



Figure 14. Skein relation for full links plane projection

- Now take $\mathcal{M}=S^{3}$ and denote the partition function of $n$ unlinked, unknotted loops living on the three sphere $S^{3}$ as $Z\left(S^{3} ; C_{1}, \ldots, C_{n}\right)$. Following Figure 14, then (6.12) sum up to

$$
\alpha Z\left(S^{3} ; C\right)+\beta Z\left(S^{3} ; C^{2}\right)+\gamma Z\left(S^{3} ; C\right)=0 .
$$

Using the factorization properties earlier outlined, then

$$
\alpha \frac{Z\left(S^{3} ; C\right)}{Z\left(S^{3} ; C\right)}+\beta \frac{Z\left(S^{3} ; C^{2}\right)}{Z\left(S^{3} ; C\right)}+\gamma \frac{Z\left(S^{3} ; C\right)}{Z\left(S^{3} ; C\right)}=0
$$

implies

$$
\langle C\rangle=-\frac{\alpha+\gamma}{\beta} .
$$

- For $S U(N)_{k}$, a braiding operator $B$ whose eigenvalues are

$$
\lambda_{1}=\exp \left(\frac{i \pi(-N+1)}{N(N+k)}\right), \quad \lambda_{2}=-\exp \left(\frac{i \pi(N+1)}{N(N+k)}\right)
$$

can be used to evaluate expectation value $\langle C\rangle$ with

$$
\begin{aligned}
& \alpha=-\exp \left(\frac{2 \pi i}{N(N+k)}\right), \\
& \beta=-\exp \left(\frac{i \pi\left(2-N-N^{2}\right)}{N(N+k)}\right)+\exp \left(\frac{i \pi\left(2+N-N^{2}\right)}{N(N+k)}\right), \\
& \gamma=\exp \left(\frac{2 \pi i\left(1-N^{2}\right)}{N(N+k)}\right) .
\end{aligned}
$$

- In fact, by introducing variable $q=\exp \left(\frac{2 \pi i}{N+k}\right)$, the very property of Jones polynomial

$$
-q^{N / 2} L+\left(q^{1 / 2}-q^{-1 / 2}\right) L^{\prime}+q^{-N / 2} L^{\prime \prime}=0
$$

realizes the expectation value for the single loop as

$$
\langle C\rangle=-\frac{\alpha+\gamma}{\beta}=\frac{q^{N / 2}-q^{-N / 2}}{q^{1 / 2}-q^{-1 / 2}},
$$

- While this computation is for a single Wilson loop, the same procedure generalizes to more many complicated loops. This immediately suggest the interpretation of the partition function $Z(M ; L, R)$ of the $S U(N)_{k}$ Chern-Simons theory as nothing but the Jones polynomial, evaluated at $q=\exp \left(\frac{2 \pi i}{N+k}\right)$, and the problem is solved. 


\section{Conclusion}

Our study cut across many interesting physical ideas, each of which are very enlightening and may worth studying independently. These include some progresses in planar condensed matter physics leading to non-Abelian anyons, and then to finding a good topological quantum field theory (TQFT) to describe those physics. While the studies in relation to understanding these rich concepts is inexhaustive, we have been able to, at least, achieve a primary aim of recognizing the $2+1$-dimensional Chern-Simons gauge theory as a good example TQFT exhibiting most important features of the underlying physics of the quantum hall system and the non-Abelian anyons.

The study also exposed us to the realization that, there is really lots of stories and ideas about quantum Chern-Simons invariants in topology, and physics. Indeed, we have learnt a very important lesson, in that, given a link $L=\cup_{C_{i}} \subset S^{3}$ in the representation $R_{i}$ of the $S U(N)$ Chern-Simons level $k$ theory, the partition function,

$$
Z\left(S^{3} ; L, R_{i}\right)=\int \mathcal{D} a \exp \left(\frac{i k}{4 \pi} \int_{S^{3}} \operatorname{Tr}\left(a \times d a+\frac{2}{3} a \times a \times a\right)\right) \prod_{i} \operatorname{Tr}_{R_{i}} \mathcal{P} \exp \int_{C_{i}} a,
$$

is the Jones polynomial evaluated at variable $q=\exp \left(\frac{2 \pi i}{N+k}\right)$. This is Witten's novel achievement. As such, it has provided another exchange between mathematics and physical studies.

Suffices to mention here that the Chern-Simons terms have been accommodated into some other theories. Due to the gauge principle it exhibits, CS effective modification connects interesting areas of physics studies such as particle physics, String Theory Loop Quantum Gravity and Cosmology. There is so much more to groak.

\section{Acknowledgments}

I thank Richard Eager for making this topic available for my study and seminar work. My knowledge of the topic improves greatly through some useful discussion with Ingmar Saberi.

\section{References}

[1] Edward Witten, Quantum field theory and the Jones polynomial, Comm. Math. Phys. 121(3) (1989) 351-399

[2] D. C. Tsui, H. L. Stormer, and A. C. Gossard, Two-Dimensional Magnetotransport in the Extreme Quantum Limit, Phys.Rev.Lett. 48 (1982) 1559-1562

[3] Edwin Hall, On a New Action of the Magnet on Electric Currents, American Journal of Mathematics 2(3) (1879) 287-292

[4] R. B. Laughlin, Anomalous Quantum Hall Effect: An Incompressible Quantum Fluid with Fractionally Charged Excitations, Phys. Rev. Lett. 50 ( 1983) 1395-1398

[5] J.M. Leinaas and J. Myrheim, On the theory of identical particles, Nuovo Cim B (1977) 37

[6] B. I. Halperin, Statistics of Quasiparticles and the Hierarchy of Fractional Quantized Hall States, Phys. Rev. Lett. 52 ( 1984) 1583-1586

[7] Frank Wilczek, Magnetic Flux, Angular Momentum, and Statistics, Phys.Rev.Lett. 48 (1982) $1144-1146$ 
[8] Frank Wilczek, Quantum Mechanics of Fractional-Spin Particles, Phys. Rev. Lett. 49 (1982) 957-959

[9] F. D. M. Haldane, Fractional Quantization of the Hall Effect: A Hierarchy of Incompressible Quantum Fluid States, Phys. Rev. Lett. 51 (1983) 605-608; Martin Greiter, Microscopic formulation of the hierarchy of quantized Hall states, Physics Letters B 336 (1994) 48-53 .

[10] E. Artin, Theorie der Zöpfe, Abh. Math. Sem. Univ. Hamburg 4 (1926), 47-72, FdM51, 450; E. Artin, Theory of Braids, Ann. of Math. 48, no. 1 (1947a) 101-126

[11] J.K. Slingerland and F.A.Bais, Quantum groups and non-Abelian braiding in quantum Hall systems, Nuclear Physics B 612 (2001) 229-290 ; Simon Trebst et. al. A short introduction to Fibonacci anyon models, Prog Theor Phys (2008) 176: 384-407

[12] A. Zee, Quantum hall fluids, Field Theory, Topology and Condensed Matter Physics, LNP 456 (1994) 99-153

[13] S. Chern, J. Simons, Characteristic forms and geometric invariants, Ann. of Math. 99 (1974) 48-69; Daniel S. Freed, Remarks on Chern-Simons theory, Bull. Amer. Math. Soc. 46 (2009), 221

[14] Xiao-Gang Wen, Topological orders and edge excitations in fractional quantum Hall states, Advances in Physics 44 (1995) 405-473

[15] E.Guadagnini et. al., Wilson lines in Chern-Simons theory and link invariants, Nuclear Physics B $330(1990) 575$

[16] V.F.R Jones, Polynomial invariant for knots via von Neumann algebras, Bull. Amer. Math. Soc. (N.S.) 12 (1985) 1, 103-111 ; L. H. Kauffman State models and the Jones polynomial, Topology 26 (1987) 3, 395-407 ; L. H. Kauffman, Knots and Physics, World Scientific, 3 (2001)

[17] Poul Heegaard, Forstudier til en topologisk Teori for de algebraiske Fladers Sammenhang, Thesis (1898) JFM 29.0417 .02 ; Jesse Johnson, Notes on Heegaard Splittings, JesseJ

[18] Michael Atiyah, Topological quantum field theory, Publ. Math. IHES 68 (1989) 175-186; John C. Baez, Quantum Quandaries: a Category-Theoretic Perspective, arXiv: quant-ph/0404040

[19] Erik Verlinde, Fusion rules and modular transformations in $2 D$ conformal field theory, Nucl. Phys. B300 (1988) 360; R. Dijkgraaf and E. Verlinde, Modular Invariance and the Fusion Algebra, Nucl. Phys. B (Proc. Suppl.) 5B (1988) 87; M. Blau and G. Thompson, Derivation of the Verlinde Formula from Chern-Simons Theory and the G/G model, Nucl.Phys. B408 (1993) 345

\section{Complementary Reading}

- David Tong, The Quantum Hall Effect, TIFR Infosys Lectures, arXiv:1606.06687

- Jiannis K. Pachos, Introduction to Topological Quantum Computation, CUP (2012)

- Chetan Nayak et. al., Non-Abelian Anyons and Topological Quantum Computation, Rev. Mod. Phys. 80, 1083 (2008)

- W. B. Raymond Lickorish, An Introduction to Knot Theory, Springer (1997) 